\title{
First report on the occurrence of Rickettsia slovaca and Rickettsia raoultii in Dermacentor silvarum in China
}

\author{
Zhan-Cheng Tian, Guang-Yuan Liu*, Hui Shen, Jun-Ren Xie, Jin Luo and Mei-Yuan Tian
}

\begin{abstract}
Background: Rickettsioses are among both the longest known and most recently recognized infectious diseases. Although new spotted fever group rickettsiae have been isolated in many parts of the world including China, Little is known about the epidemiology of Rickettsia pathogens in ticks from Xinjiang Autonomous Region of China.

Methods: In an attempt to assess the potential risk of rickettsial infection after exposure to ticks in Xinjiang Uygur Autonomous Region of China, a total of 200 Dermacentor silvarum ticks collected in Xinyuan district were screened by polymerase chain reaction based on the outer membrane protein A gene.

Results: 22 of the 200 specimens (11\%) were found to be positive by PCR. Phylogenetic analysis of OmpA sequences identified two rickettsial species, Rickettsia raoultii (4.5\%) and Rickettsia slovaca (6.5\%).

Conclusions: This study has reported the occurrence of Rickettsia raoultii and Rickettsia slovaca in Xinjiang Autonomous Region of China and suggests that Dermacentor silvarum could be involved in the transmission of rickettsial agents in China. Further studies on the characterization and culture of rickettsial species found in Dermacentor silvarum should be performed to further clarify this. Additionally, the screening of human specimens for rickettsial disease in this region will define the incidence of infection.
\end{abstract}

\section{Background}

Tick-transmitted diseases are a focus of increasing medical interest worldwide. Ticks are the main vectors and reservoirs of rickettsial pathogens responsible for spotted fever. Rickettsioses are among both the longest known and most recently recognized infectious diseases. The clinical features include fever, headache, eruption, and incidental eschar formation at the site of tick bites [1]. The etiological agents belonging to the genus Rickettsia are currently divided into two groups: the typhus group and the spotted fever group. The latter group includes an increasing number of newly identified species.

In China, many spotted fever group (SFG) rickettsiae belong to $R$. sibirica, including 2 subspecies, i.e., $R$. sibirica sibirica, the agent of North Asian tick typhus

\footnotetext{
* Correspondence: liuguangyuan2002@sina.com

State Key Laboratory of Veterinary Etiological Biology, Key Laboratory of Veterinary Parasitology of Gansu Province, Lanzhou Veterinary Research Institute, Chinese Academy of Agricultural Sciences, Lanzhou, Gansu Province 730046, People's Republic of China
}

(c) 2012 Tian et al; licensee BioMed Central Ltd. This is an Open Access article distributed under the terms of the Creative Commons Attribution License (http://creativecommons.org/licenses/by/2.0), which permits unrestricted use, distribution, and reproduction in any medium, provided the original work is properly cited. detected in Dermacentor silvarum and D. sinicus in northern China, and R. sibirica mongolotimonae, the agent of lymphangitis-associated rickettsiosis isolated from Hyalomma asiaticum in Inner Mongolia [2,3]. Rickettsia heilongjiangensis, first isolated from D. silvarum ticks in Heilongjiang Province, can cause spotted fever in humans $[4,5]$. Rickettsia hulinii was first isolated from Haemaphysalis concinna in Heilongjiang Province, but its pathogenic role in humans has not been demonstrated [6]. However, there is limited information on the epidemiology of rickettsial species in ticks from the Xinjiang Uygur Autonomous Region (XUAR), China, apart from a case report of a SFG rickettsia from a patient in XUAR [7].

In the present study, we assessed the prevalence of rickettsial pathogens in D. silvarum from Xinyuan district, XUAR using molecular techniques. Identification and characterization of these circulating agents is crucial for the development of preventive measures in response to the gradually increasing exposure of humans to tick vectors.

\section{Biomed Central}




\section{Methods}

\section{Ticks and DNA extraction}

A total of 200 adult female ticks were identified as $D$. silvarum based on morphological characteristics [8]. Briefly, the ticks were disinfected in $70 \%$ ethanol for 10 min, rinsed with sterilized distilled water, placed in a microtube, and mechanically disrupted with sterile scissors in $50 \mu \mathrm{l}$ of DNA extraction buffer (10 mM Tris $\mathrm{pH}$ 8.0, 2 mM EDTA, 0.1\% sodium dodecyl sulfate, and 500 $\mu \mathrm{g}$ of proteinase $\mathrm{K}$ per $\mathrm{ml}$ ). The sample was incubated at $56^{\circ} \mathrm{C}$ for $4 \mathrm{hr}$, then boiled at $100^{\circ} \mathrm{C}$ for $10 \mathrm{~min}$ to inactivate the proteinase $\mathrm{K}$. After centrifugation, the supernatant was transferred to a fresh microtube and DNA was purified by extracting twice with an equal volume of phenol-chloroform, precipitated in ethanol and the DNA resuspended in $20 \mu \mathrm{l}$ elution buffer, which was then stored at $-20^{\circ} \mathrm{C}$ until used.

\section{PCR amplification and sequence analysis of ompA} PCR reactions were performed using primers Rr190.70p and Rr190.602n (5'-ATGGCGAATATTTCTCCAAAA3'; 5'-AGTGCAGCATTCGCTCCCCCT-3') designed to amplify the outer membrane protein A (ompA) gene of rickettsial species as described previously [9]. Distilled water instead of tick DNA template was used as a negative control. PCR products were purified and sequenced. These were compared with previously published sequences deposited in GenBank using BLAST. Partial ompA sequences of rickettsial species were aligned with that of 27 rickettsial species by the Clustal W program with default parameter settings (DNAStar version 4.01, Madison, WI, USA). Outer membrane protein P44 from Anaplasma phagocytophila (AF412830) was used as an outlier group in the alignments of nucleotide sequences of ompA. A phylogenetic tree was constructed using the Kimura 2-parameter model and the neighbour-joining algorithm of MEGA 4.0 software [10].

\section{Results}

PCR products of the rickettsial ompA gene with expected size (530-533 bp) were amplified from $D$. silvarum ticks. Sequencing data of the 22 positive samples indicated two distinct rickettsial species from the 200 ticks screened. Nine of these were identified as $R$. raoutii and the remaining 13 were $R$. slovaca. Six of the $R$. raoutii samples were $100 \%$ identical to each other but exhibited 99.1-99.8\% (530/530) variability with the remaining $3 R$. raoutii samples. However, all 9 samples of $R$. raoutii were $99.8-100 \%$ and $99.2-99.4 \%$ (511/511) homologous with the $R$. raoultii Marne and Khabarovsk strains respectively. Of the $13 R$. slovaca samples identified, 11 were $100 \%$ identical, while the remaining 2 were slightly divergent with a 99.6 and $99.8 \%(533 / 533)$ homology. Collectively all the $R$. slovaca samples were 99.8-100\% (533/533) homologous with the $R$. slovaca (HM161798.1) strain. The variability between the two rickettsial species identified from the ticks was $5.5-5.8 \%$. All of the unique sequencing data (not including identical sequences) were deposited in GenBank with accession numbers JN400401-JN400407.

Phylogenetic analysis indicated the formation of 3 clades within the rickettsial neighbour-joining tree (Figure 1). All of $R$. slovaca like samples formed a single clade with $R$. slovaca while the $R$. raoutii like samples formed two clades. One of these clades was closely related to the $R$. raoutii reference strains and the other formed a unique $R$. raoutii clade which is possibly a new strain.

\section{Discussion}

The present study reports for the first time the occurrence of $R$. raoultii and $R$. slovaca in D. silvarum ticks in China. Rickettsia raoultii has been reported in Dermacentor ticks in Europe and Russia, including strains KhabarovskT, Marne, Shayman, 8/9 Karaganda and Elanda-23/95 [11]. Until the present study $R$. raoultii had not been definitely reported in China and only two ompA gene sequences from two isolates from Jilin province had previously been deposited in GenBank (AY093696.1 and DQ188831.1), however, this was not reported in the literature. The rickettsial strains identified in this study were defined by using published phylogenetic classifications of rickettsial species [3,6]. PCR and sequencing of rickettsial ompA genes, identified $R$. raoultii in $4.5 \%$ of $D$. silvarum ticks collected in XUAR. Furthermore, phylogenetic analysis revealed that the positive samples detected formed a distinct clade with $R$. raoultii with a high (91) bootstrap value, which included strains KhabarovskT and Marne. Of the nine $R$. raoutii samples identified in this study, eight were from Xinyuan and one was from Gansu province. Together with the two samples from Jilin province (AY093696.1 and DQ188831.1). This suggests that $R$. raoultii has a geographical spread that encompasses a large area of China.

Rickettsia slovaca as a human pathogen [12] was first isolated in 1968 from a Dermacentor marginatus tick in Slovakia [13]. Since then, it has been found in both $D$. marginatus and $D$. reticulatus ticks from Western Europe and central Asia [14-18]. The rickettsial disease caused by $R$. slovaca is called tick-borne lymphadenopathy (TIBOLA) or Dermacentor-borne necrosiserythema-lymphadenopathy $[19,20]$ and its epidemiological pattern and clinical features in patients from France, Hungary and Spain have been investigated $[18,21]$. It therefore seems to closely follow the 


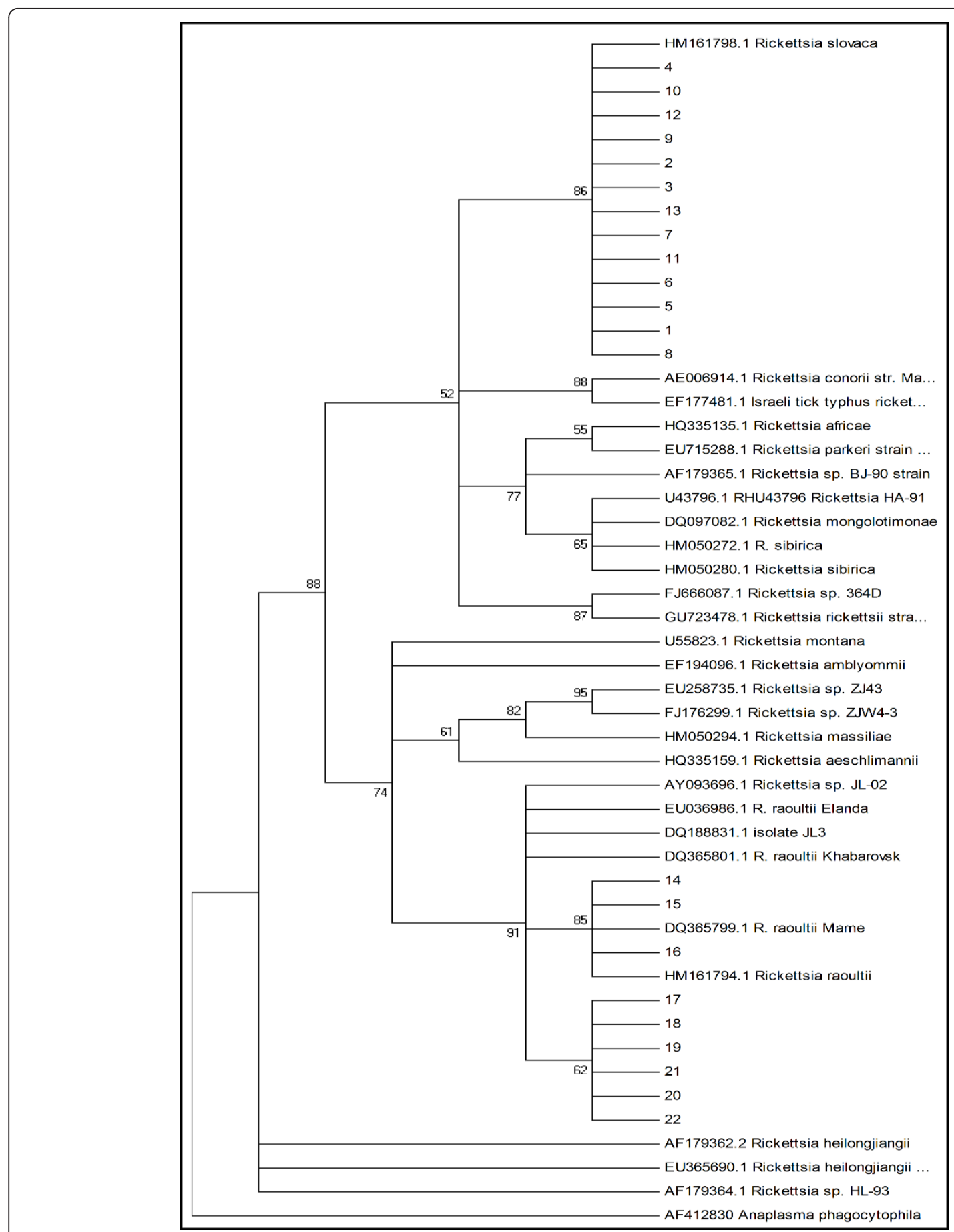

Figure 1 Constructed phylogenetic tree based on partial ompA gene sequences from rickettsial positive tick samples using neighbour-joining method with 1,000 bootstraps and a cut off value of $50 \%$.

distribution of its main host tick, $D$. marginatus, which can be found throughout Europe and as far as the western border of China [15]. However, in the present study, we detected R. slovaca by PCR in $6.5 \%$ of $D$. silvarum ticks collected from XUAR. The results of the present study identified $R$. slovaca in the D. silvarum ticks which could be transmitted to humans and cause disease. Further studies on the characterization and culture of rickettsial endosymbionts found in D. silvarum collected in XUAR should be performed. 


\section{Conclusions}

This is the first report of $R$. raoultii and $R$. slovaca in China and suggests that $D$. silvarum could be involved in the transmission of $R$. slovaca in China. Further studies on the characterization and culture of rickettsial endosymbionts found in D. silvarum from XUAR should be performed.

\section{Acknowledgements}

The authors thank Prof. Una Ryan and Prof. Xing-Quan Zhu for improving the manuscript. We also thank the anonymous reviewers for their helpful comments and suggestions. This work was supported, in part, by the Natural Science Foundation of Gansu Province, China (No.096RJZA128) and Hi-Tech Research and Development Program of China (No. 2009AA10Z402).

\section{Authors' contributions}

LGY and TZC conceived and designed the study, and critically revised the manuscript. TZC, SH and LJ performed the experiments, analysed the data and drafted the manuscript. XJR and TMY helped in study implementation and data collection. All authors read and approved the final manuscript.

\section{Competing interests}

The authors declare that they have no competing interests.

Received: 21 September 2011 Accepted: 19 January 2012

Published: 19 January 2012

\section{References}

1. Kaabia N, Letaief $A$ : Characterization of rickettsial diseases in a hospitalbased population in central Tunisia. Ann N Y Acad Sci 2009, 1166:167-171.

2. $Y u$ XJ, Fan MY, Xu G, Liu Q, Raoult D: Genotypic and antigenic identification of two new strains of spotted fever group rickettsiae isolated from China. J Clin Microbiol 1993, 31:83-88.

3. Zhang L, Jin J, Fu X, Raoult D, Fournier PE: Genetic differentiation of Chinese isolates of Rickettsia sibirica by partial 154 ompA gene sequencing and multispacer typing. J Clin Microbiol 2006, 44:2465-2467.

4. Fournier PE, Suhre K, Fournous G, Raoult D: Estimation of prokaryote genomic DNA G+C content by sequencing universally conserved genes. Int J Syst Evol Microbiol 2006, 56:1025-1029.

5. Jiao Y, Wen B, Chen M, Niu D, Zhang J, Qiu L: Analysis of immunoprotectivity of the recombinant OmpA of Rickettsia heilongjiangensis. Ann N Y Acad Sci 2005, 1063:261-265.

6. Zhang JZ, Fan MY, Wu YM, Fournier PE, Raoult D: Genetic classification of "Rickettsia heilongjiangii" and "Rickettsia hulinii," two Chinese spotted fever group rickettsiae. J Clin Microbiol 2000, 38:3498-3501.

7. Fan MY, Wang JG, Jiang YX, Zong DG, Brenda L, David HW: Isolation of a Spotted Fever Group Rickettsia from a Patient and Related Ecologic Investigations in Xinjiang Uygur Autonomous Region of China. J Clin Microbiol 1987, 25:628-632.

8. Voltsit OV: Sexual dimorphism of the nymphal phase of ixodid tick species in the genus Dermacentor. Parazitologiia 1989, 23:20-28.

9. Regnery RL, Spruill CL, Plikaytis BD: Genotypic identification of rickettsiae and estimation of intraspecies sequence divergence for portions of two rickettsial genes. J Bacteriol 1991, 173:1576-1589.

10. Tamura K, Dudley J, Nei M: MEGA4: molecular evolutionary genetics analysis (MEGA) software version 4.0. Mol Biol Evol 2007, 24:1596-1599.

11. Mediannikov O, Matsumoto K, Samoylenko I, Drancourt M, Roux VR, Rydkina E, Davoust B, Tarasevich I, Brouqui P, Fournier PE: Rickettsia raoultii sp. nov., a spotted fever group rickettsia associated with Dermacentor ticks in Europe and Russia. Int J Syst Evol Microbiol 2008, 58:1635-1639.

12. Cazorla C, Enea M, Lucht F, Raoult D: First isolation of Rickettsia slovaca from a patient, France. Emerg Infect Dis 2003, 9:135.

13. Rehacek J: Rickettsia slovaca, the organism and its ecology. Acta SC Nat Brno 1984, 18:1-50

14. Balayeva NM, Eremeeva ME, Raoult D: Genomic identification of Rickettsia slovaca among spotted fever group rickettsia isolates from Dermacentor marginatus in Armenia. Acta Virol 1994, 38:321-325.
15. Eremeeva ME, Balayeva NM, Ignatovich VF, Raoult D: Proteinic and genomic identification of spotted fever group rickettsiae isolated in the former USSR. J Clin Microbiol 1993, 31:2625-2633.

16. Beati L, Finidori JP, Raoult D: First isolation of Rickettsia slovaca from Dermacentor marginatus in France. Am J Trop Med Hyg 1993, 48:257-268.

17. Beati L, Humair PF, Aeschlimann A, Raoult D: Identification of spotted fever group rickettsiae isolated from Dermacentor marginatus and Ixodes ricinus ticks collected in Switzerland. Am J Trop Med Hyg 1994, 51:138-148.

18. Raoult D, Lakos A, Fenollar F, Beytout J, Brouqui P. Fournier PE: Spotless rickettsiosis caused by Rickettsia slovaca and associated with Dermacentor ticks. Clin Infect Dis 2002, 34:1331-1334.

19. Lakos A: Tick-borne lymphadenopathy (TIBOLA)-a new, probably rickettsial infection. Inf Dis Rev 1999, 1:114-116.

20. Oteo JA, Ibarra V: DEBONEL (Dermacentor-bornenecrosis-erythemalymphadenopathy). Una nueva enfermedad transmitida por garrapatas? Enferm Infecc Microbiol Clin 2002, 20:51-52.

21. Oteo JA, Ibarra V, Blanco JR, Martínez DAV, Márquez FJ, Portillo A, Raoult D, Anda P: Dermacentor-borne necrosis erythema and lymphadenopathy: clinical and epidemiological features of a new tick-borne disease. Clin Microbiol Infect 2004, 10:327-331.

doi:10.1186/1756-3305-5-19

Cite this article as: Tian et al:: First report on the occurrence of Rickettsia slovaca and Rickettsia raoultii in Dermacentor silvarum in China. Parasites \& Vectors 2012 5:19

\section{Submit your next manuscript to BioMed Central and take full advantage of:}

- Convenient online submission

- Thorough peer review

- No space constraints or color figure charges

- Immediate publication on acceptance

- Inclusion in PubMed, CAS, Scopus and Google Scholar

- Research which is freely available for redistribution
C Biomed Central 